\title{
Photoluminescence from an individual double-walled carbon nanotube
}

\author{
Dmitry I. Levshov, ${ }^{1,2}$ Romain Parret, ${ }^{1}$ Huy-Nam Tran, ${ }^{1}$ Thierry Michel, ${ }^{1}$ Thi Thanh Cao, ${ }^{3}$ Van Chuc Nguyen, ${ }^{3}$ Raul Arenal, ${ }^{4,5}$ \\ Valentin N. Popov, ${ }^{6}$ Sergei B. Rochal, ${ }^{2}$ Jean-Louis Sauvajol, ${ }^{1}$ Ahmed-Azmi Zahab,${ }^{1}$ and Matthieu Paillet ${ }^{1, *}$ \\ ${ }^{1}$ Laboratoire Charles Coulomb (UMR5221), CNRS-Université de Montpellier, F-34095 Montpellier, France \\ ${ }^{2}$ Faculty of Physics, Southern Federal University, Rostov-on-Don, Russia \\ ${ }^{3}$ Institute of Materials Science, Vietnam Academy of Science and Technology, 18 Hoang Quoc Viet, Hanoi, Vietnam \\ ${ }^{4}$ Instituto de Nanociencia de Aragón, Campus Rìo Ebro, Edificio I+D. C/Mariano Esquillo, 50018 Zaragoza, Spain \\ ${ }^{5}$ ARAID Foundation, 50018 Zaragoza, Spain \\ ${ }^{6}$ Faculty of Physics, University of Sofia, 5 James Bourchier Boulevard, 1164 Sofia, Bulgaria \\ (Received 23 May 2017; revised manuscript received 28 July 2017; published 7 November 2017)
}

\begin{abstract}
We report direct and unambiguous evidence of the existence of inner semiconducting tube (ISCT) photoluminescence (PL) from measurements performed on four individual freestanding index-identified double-walled carbon nanotubes (DWNTs). Based on thorough Rayleigh scattering, Raman scattering, and PL experiments, we are able to demonstrate that the ISCT PL is observed with a quantum yield estimated to be a few $10^{-6}$ independent of the semiconducting or metallic nature of the outer tube. This result is mainly attributed to ultrafast exciton transfer from the inner to outer tube. Furthermore, by carrying out PL excitation experiments on the $(14,1) @(15,12)$ DWNT, we show that the ISCT PL can be detected through the optical excitation of the outer tube, indicating that the exciton transfer can also occur in the opposite way.
\end{abstract}

DOI: 10.1103/PhysRevB.96.195410

\section{INTRODUCTION}

Double-walled carbon nanotubes (DWNTs), consisting of two coaxial and weakly van der Waals coupled single-walled carbon nanotubes (SWNTs), are one of the most ideal and fundamental systems to study the effects of interwall coupling on the physical properties in one-dimensional structures. The properties of DWNTs are related to (i) the individual nature of the constituent SWNTs, which can be either metallic (M) or semiconductor (SC), (ii) the van der Waals interactions, depending on the wall-to-wall distance, typically ranging from 0.3 to $0.4 \mathrm{~nm}[1-6]$, and (iii) the matching of the atomic lattices of inner and outer tubes (moiré patterns), which plays a decisive role in determining the electronic structure of DWNTs even without any commensurability [7,8]. It has even been shown recently that the mechanical interlayer coupling steers their structural properties and favors certain configurations [9].

Regarding potential applications, e.g., in photonics or biology, a key property is inner semiconducting tube (ISCT) photoluminescence (PL) [10]. In DWNTs, the inner tube possesses a special status, granted by the outer tube, which acts as a shield and thus provides higher mechanical, thermal, and chemical stability even in aggressive environments compared to SWNTs [11-13]. However, the ability of an ISCT to exhibit PL is still a subject of debate, and no consensus has been reached so far. For instance, a rather strong PL signal was observed in different investigations [14-19], whereas other studies established its (quasi)nonexistence [20,21]. Two reasons can explain the origin of these contradicting conclusions.

(i) The difficulty in measuring a PL signal in DWNTs could be related to an intrinsic low PL quantum yield (QY) of the ISCT due to the interactions between inner and outer layers [22-24], especially exciton energy transfer [21,25] and charge transfer [26]. Supporting the latter hypothesis, the PL enhancement from the $(8,3)$ ISCT filled with linear carbon

\footnotetext{
*Corresponding author: matthieu.paillet@umontpellier.fr
}

chains has recently been demonstrated and understood in terms of charge transfer from the ISCT to the carbon chains, which counterbalances the quenching mechanism of PL induced by the outer tube [27].

(ii) Up to now, the PL experiments have been performed on suspensions of ensembles of individual DWNTs. In such samples, it is unclear whether the observed PL signal originates from the ISCT $[14,18,19,28]$ or from SWNT impurities present in the starting materials or originating from inner tubes extracted from outer tubes during the suspension preparation process [11,20,21,29]. This highlights the difficulties of studies conducted on ensembles and emphasizes the call for investigations at the single-DWNT level. Combining electron diffraction (ED) and Raman/optical spectroscopy to examine individual SWNTs appeared to be the most direct and unambiguous method to address the relationship between their structure and physical properties [30-33]. Recently, the latter approach has allowed several advances regarding the understanding of DWNT physical properties, especially regarding the effect of the interwall coupling on phonons and electronic states $[4,6,7,34,35]$.

We report here direct and unambiguous evidence of the existence of ISCT PL from measurements performed on four individual freestanding index-identified DWNTs (three SC@SC and one SC@M). On the basis of thorough Rayleigh scattering, Raman scattering, PL, and PL excitation (PLE) experiments, we are able to demonstrate that the PL of the ISCT of DWNTs is observed for both SC and M outer tubes. The PL features, including its QY, are discussed and compared to those of SWNTs.

The experimental information is described in Sec. II. The results are presented in Sec. III and discussed in Sec. IV.

\section{SAMPLE AND EXPERIMENTAL METHODS}

Ultralong individual DWNTs were grown using the catalytic chemical-vapor deposition (CCVD) method directly 


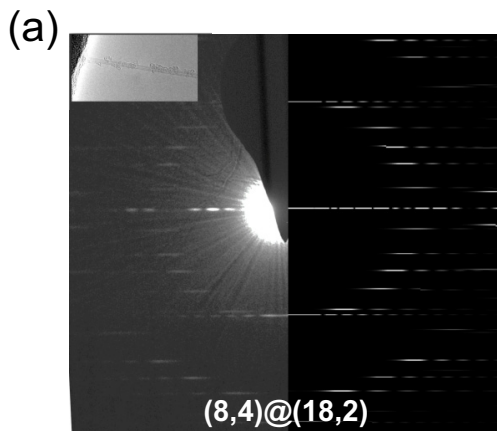

(b)

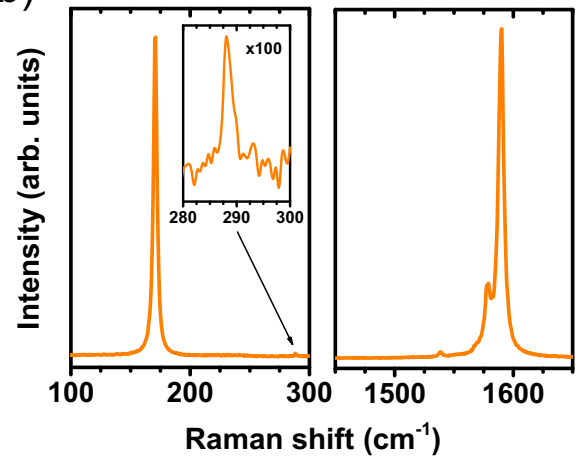

(c)

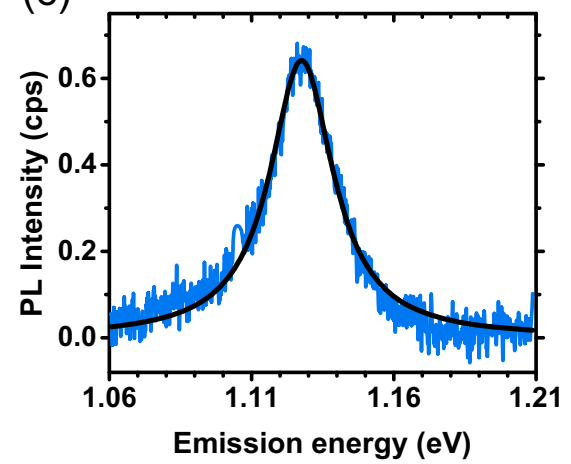

FIG. 1. The $(8,4) @(18,2)$ DWNT. (a) ED pattern, experimental (left part) and calculated (right part). The inset shows an HRTEM image of this DWNT. (b) RBLM (left) and $G$ (right) mode ranges of the Raman spectrum excited at $2.21 \mathrm{eV}$. The inset in the left panel shows a close-up of the out-of-phase RBLM. (c) PL emission spectrum excited at $2.21 \mathrm{eV}$. The solid blue line shows the experimental data, and the solid black line shows the Lorentzian fit.

across open-slit structures $\left(50 \times 1000 \mu \mathrm{m}^{2}\right)$ fabricated on silicon substrates. The details of the synthesis of individual DWNTs are given in the Supplemental Material [36].

The DWNTs were characterized by high resolution transmission electron microscopy (HRTEM) and ED and investigated using Raman, Rayleigh and PL measurements [36]. A DWNT structure is uniquely defined by the chiral indices $\left(\mathrm{n}_{i}\right.$, $\left.\mathrm{m}_{i}\right)$ and $\left(\mathrm{n}_{o}, \mathrm{~m}_{o}\right)$ of its constituent inner and outer layers, respectively [37]. Such DWNT is denoted as $\left(\mathrm{n}_{i}, \mathrm{~m}_{i}\right) @\left(\mathrm{n}_{o}, \mathrm{~m}_{o}\right)$. Here, the structure identification procedure follows the one described in Ref. [38] and is detailed in [36].

HRTEM images and ED patterns were recorded in a FEI Titan Cube microscope (equipped with a spherical aberration corrector) operating at $80 \mathrm{kV}$ and within short acquisition times (less than $5 \mathrm{~s}$ for ED) to reduce damage induced by electron diffraction.

Rayleigh spectra of individual freestanding DWNTs were measured in a backscattering geometry using a crosspolarization scheme with a Fianium supercontinuum laser as a light source and a fiber-fitted QE-Pro Ocean Optics spectrometer for detection. Resonant Raman scattering measurements were carried out using an iHR550 Jobin-Yvon spectrometer (1800 gratings or 1200 grooves $/ \mathrm{mm}$ ) equipped with a liquidnitrogen-cooled, back-illuminated silicon charge-coupled device detector in a micro-Raman backscattering configuration. The home-made microscope is equipped with a $100 \times$ objective lens (numerical aperture of 0.95). The samples were mounted on a three-axis piezo-electric stage (PIMars P-563, Physik Instrumente) to ensure precise positioning and focusing of the laser spot. Incident excitations from various continuous-wave lasers were used: $\mathrm{HeNe}$ at $632.8 \mathrm{~nm}(1.96 \mathrm{eV})$, diode-pumped solid-state lasers at $457 \mathrm{~nm}(2.71 \mathrm{eV}), 532 \mathrm{~nm}(2.33 \mathrm{eV})$, and $561 \mathrm{~nm}(2.21 \mathrm{eV})$, and a Ti:sapphire laser filtered using a tunable laser line filter [39] in the near infrared. To avoid heating effects, the laser intensity impinging the sample was kept below $100 \mathrm{~kW} / \mathrm{cm}^{2}$. Photoluminescence measurements were performed on the same setup but using a liquid-nitrogen-cooled InGaAs detector and a grating blazed at $1 \mu \mathrm{m}$ with 300 grooves $/ \mathrm{mm}$. In all the Raman and PL measurements, the incident light polarization is along the nanotube axis.

\section{RESULTS}

The first investigated SC@SC DWNT is unambiguously identified as $(8,4) @(18,2)$ from its ED pattern [Fig. 1(a)] and its Raman spectrum measured at $2.21 \mathrm{eV}$ [561 nm; Fig. 1(b)]. The PL emission spectrum obtained by exciting this DWNT at $2.21 \mathrm{eV}$ is shown in Fig. 1(c). The PL peak is centered at $1.128 \mathrm{eV}(1099 \mathrm{~nm})$. Its linewidth (FWHM) is $\approx 27 \mathrm{meV}$, and its maximum intensity is around 0.6 counts/s for a laser power impinging the tube $P_{\text {laser }}$ of $60 \mu \mathrm{W}$. The observed PL peak is attributed to the emission from the first excitonic transition of the ISCT $\left(S_{11}^{i}\right)$, since its position is close to the one expected around $1.15 \mathrm{eV}$ [40] for a $(8,4) \mathrm{SWNT}$. Regarding the excitation, the energy used $(2.21 \mathrm{eV})$ is close to both the ISCT second transition $\left(S_{22}^{i}\right)$ and the outer tube third transition $\left(S_{33}^{o}\right)$, predicted at 2.13 and $2.14 \mathrm{eV}$ for the $(8,4)$ and $(18,2)$ SWNTs [33], respectively. This closeness is supported by the presence of both inner- and outer-tube signatures in the Raman spectrum excited at $2.21 \mathrm{eV}$. Moreover, the observed low intensity of the high-frequency radial breathinglike mode [4,34,41] (RBLM) at $288 \mathrm{~cm}^{-1}$, dominated by the radial vibration of the $(8,4)$ ISCT, is in agreement with the theoretically predicted vanishing of the radial breathing mode (RBM) of the $(8,4)$ SWNT excited around its $S_{22}$ transition $[42,43]$.

However, it has recently been demonstrated that the optical transitions can be significantly shifted in DWNTs compared to their SWNT constituent counterparts [7]. Hence, a clear determination of the excited optical transitions requires an independent measurement of their energies. This has been achieved by measuring the Rayleigh spectra of two other individual SC@SC DWNTs, identified as $(7,6) @(16,6)$ and $(10,6) @(14,13)$ [36], which are shown in Figs. 2(a) and 2(d), respectively. By analyzing RBLM and $G$-mode resonance conditions at each excitation energy and comparing Rayleigh optical resonances to those of the constituent SWNTs [33], we have been able to assign unambiguously the resonance peaks in Rayleigh spectra to particular excitonic transitions of either inner layers $\left[S_{j j}^{i}\right.$ in Figs. 2(a) and 2(d)] or outer layers $\left[S_{j j}^{o}\right.$ in Figs. 2(a) and 2(d); see [36]].

The PL response of the $(7,6) @(16,6)$ DWNT, excited at $1.78 \mathrm{eV}$ (close to $S_{22}^{i}$ located at $1.82 \mathrm{eV}$ in the Rayleigh 
(a)

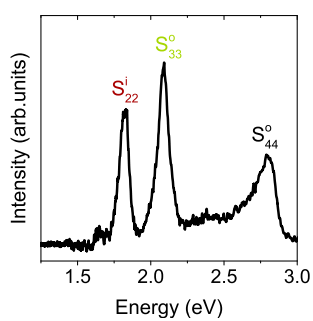

(b)
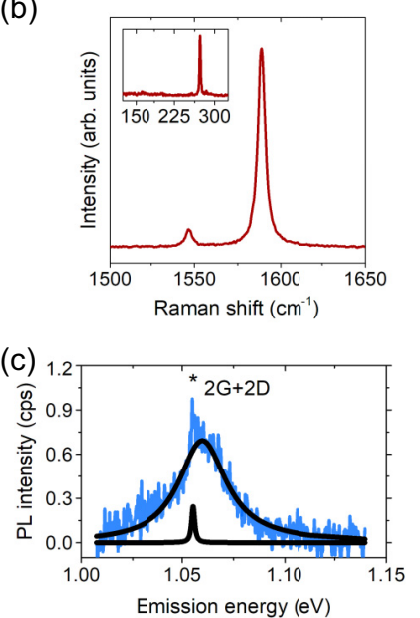

(d)

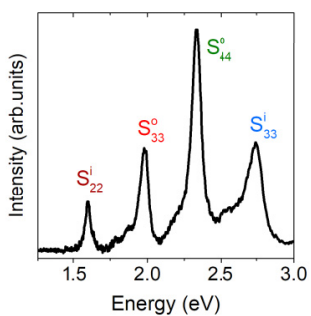

(e)
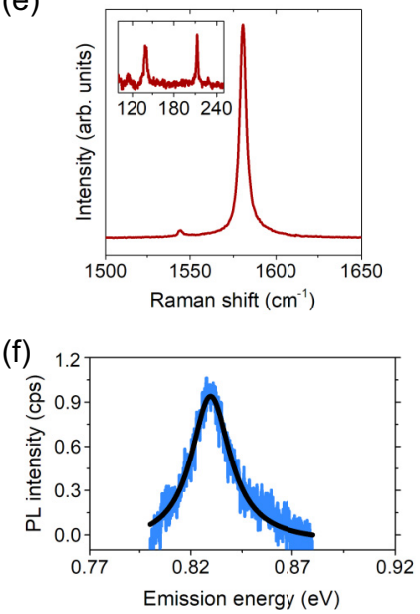

FIG. 2. The $(7,6) @(16,6)$ and $(10,6) @(14,13)$ DWNTs. (a) The $(7,6) @(16,6)$ and $(d)(10,6) @(14,13)$ Rayleigh spectra; optical transitions associated with inner (i) and outer (o) tubes are labeled on the graph as $S_{j j}^{i}$ and $S_{j j}^{o}$ respectively, where $j$ is the optical transition index. (b) The $(7,6) @(16,6)$ and $(e)(10,6) @(14,13)$ RBLM (inset) and $G$-mode ranges of Raman spectra excited at (b) 1.78 and (e) $1.6 \mathrm{eV}$ close to the $S_{22}^{i}$ optical resonances observed by Rayleigh. (c) The $(7,6) @(16,6)$ and $(\mathrm{f})(10,6) @(14,13)$ corresponding PLemission spectra excited at (c) 1.77 and (f) $1.57 \mathrm{eV}$ corresponding to $S_{22}^{i}$. Blue lines show experimental data; black lines show Lorentzian fits. The additional narrow component in the PL spectrum in (c), marked with an asterisk, is attributed to the $2 G+2 D$ Raman peak.

spectrum, with a FWHM of $36 \mathrm{meV}$ ), is shown in Fig. 2(c) and the corresponding Raman spectrum in Fig. 2(b). The PL is centered at $1.058 \mathrm{eV}(1172 \mathrm{~nm})$, its FWHM is $\approx 28 \mathrm{meV}$, and its maximum intensity is around 0.7 counts/s $\left(P_{\text {laser }} \approx 60 \mu \mathrm{W}\right)$. For comparison, $S_{11}$ of the $(7,6) \mathrm{SWNT}$ is expected at $1.13 \mathrm{eV}$ [40]. The additional narrow line in the PL spectrum [marked with an asterisk in Fig. 2(c)] is attributed to the $2 G+2 D$ combination mode [36].

For the third DWNT, $(10,6) @(14,13), S_{22}^{i}$ is located at $1.6 \mathrm{eV}(\mathrm{FWHM}=22 \mathrm{meV})$. The PL spectrum excited in resonance with this transition is shown in Fig. 2(f) [the corresponding Raman spectrum is presented in Fig. 2(e)]. In this case, the PL is centered at $0.828 \mathrm{eV}(1498 \mathrm{~nm})$, its FWHM is $\approx 24 \mathrm{meV}$, and its maximum intensity is around 1 count/s $\left(P_{\text {laser }} \approx 70 \mu \mathrm{W}\right)$. For comparison, $S_{11}$ of the $(10,6)$ SWNT is expected at $0.926 \mathrm{eV}$ [40].

Since $S_{22}^{i}$ of the $(10,6) @(14,13)$ DWNT falls in the excitation wavelength range available in our experimental setup, we carried out the measurement of the detailed PLE map in the excitation range from 1.49 up to $1.77 \mathrm{eV}$ [Fig. 3(a)]. The bright spot in the PLE map is attributed to the emission involving $S_{11}^{i}$ of the $(10,6)$ ISCT excited at its $S_{22}^{i}$.
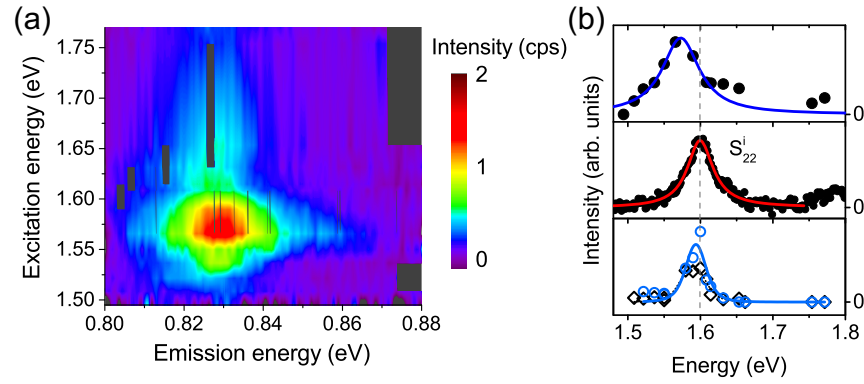

FIG. 3. The $(10,6) @(14,13)$ DWNT. (a) PLE map. Gray zones are artifacts mainly from the second order of the laser excitation line. (b) Top: PLE spectrum, integrated PL intensity as a function of laser excitation energy; black dots show experimental data, and the blue line shows the Lorentzian fit. Middle: Rayleigh spectrum; black dots show experimental data, and the red line shows the fit using an excitonic model [52]. Bottom: REPs of RBLMs, both in phase (blue open circles) and out of phase (black open diamonds), and corresponding fits using an excitonic model [52] (blue and black lines, respectively). The PLE profile fit gives a maximum of emission at an excitation energy of $1.573 \mathrm{eV}$, corresponding to $S_{22}^{i}$. The fits of the Rayleigh spectrum and REPs give the energies of this transition at 1.599 and $1.585 \mathrm{eV}$, respectively.

A fourth SC@M DWNT was unambiguously indexed from ED as $(14,1) @(15,12)$ (see [36] for a detailed characterization). The outer-tube metallic character is also clearly evidenced by the broad component in the $G$ band and the presence of electronic Raman signatures in the Raman spectra [36]. In the 1.4-1.8 eV range, two transitions are observed at 1.44 and $1.55 \mathrm{eV}$ by Rayleigh scattering [Fig. 4(c), middle panel] which are assigned to $M_{11-}^{o}$ and a superposition of $S_{22}^{i}$ and $M_{11+}^{o}$, respectively, based on the resonant Raman excitation profiles (REPs) of RBLMs [Fig. 4(b)] and $G$ modes and $M_{11-}^{o}$ electronic Raman scattering [36]. The PLE and REP maps of RBLMs of the $(14,1) @(15,12)$ DWNT in the range $1.4-1.77 \mathrm{eV}$ are presented in Figs. 4(a) and 4(b), respectively. The presence of two bright spots in the REP map [Fig. 4(b)], namely, 144 and $211 \mathrm{~cm}^{-1}$ at an excitation energy close to $1.550 \mathrm{eV}$, indicates that, as expected, both in-phase and out-of-phase RBLMs are in resonance when $S_{22}^{i}$ and $M_{11+}^{o}$ are excited simultaneously [Fig. 4(b)]. The PLE map displays a bright spot located at $0.793 \mathrm{eV}$ in emission and $1.540 \mathrm{eV}$ in excitation [Fig. 4(a)]. The emission at $0.793 \mathrm{eV}$ is assigned to the luminescence of the inner $(14,1)$ layer $\left(S_{11}^{i}\right)$. Its maximum intensity is around 2 counts/s $\left(P_{\text {laser }} \approx 70 \mu \mathrm{W}\right)$. As expected, when exciting the $M_{11-}^{o}=$ $1.44 \mathrm{eV}$ transition of the $(15,12)$ outer tube, we observe exclusively the in-phase RBLM in the Raman spectrum [single bright spot at $144 \mathrm{~cm}^{-1}$ for an excitation at $1.44 \mathrm{eV}$ in Fig. 4(b)]. Surprisingly, a slightly weaker PL signal is also detected around 0.793-1.420 eV, as shown in the PLE map in Fig. 4(a). Surprisingly, a slightly weaker PL signal is also detected around 0.793-1.420 eV, as shown in the PLE map in Fig. 4(a) (see also [36]). This shows that it is possible to measure the PL signal of the ISCT through the excitation of an optical transition of the outer tube and suggests that an exciton transfer occurs from the metallic outer tube to the ISCT. 
(a)

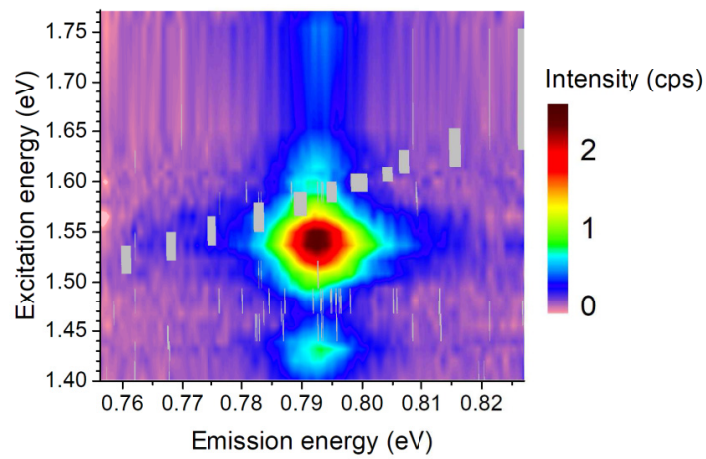

(b)

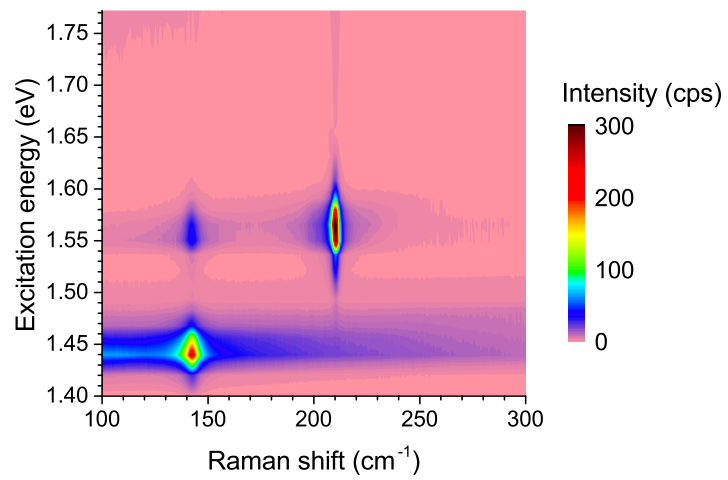

(c)

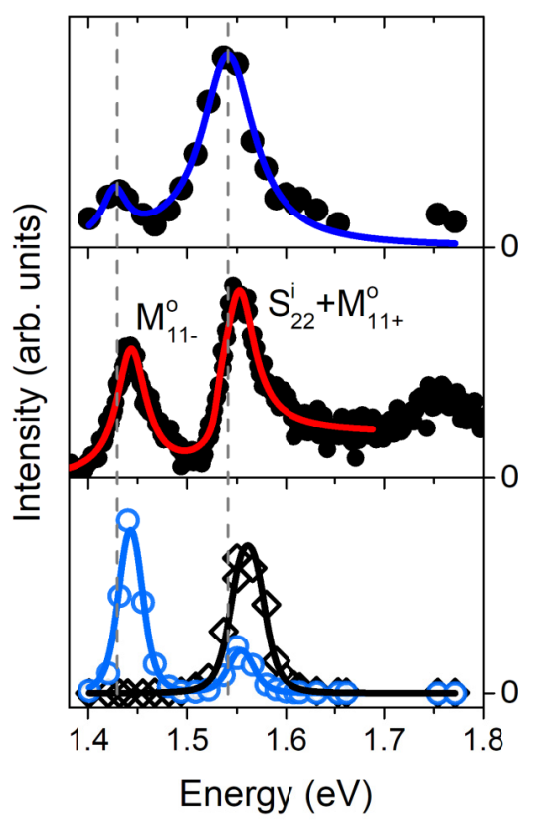

FIG. 4. The $(14,1) @(15,12)$ DWNT. (a) PL excitation map. Gray zones are artifacts mainly from the second order of the laser excitation line. (b) RBLM excitation map. (c) Top: PLE spectrum, integrated PL intensity as a function of laser excitation energy; black dots show the experimental data, and the blue line shows the Lorentzian fit. Middle: Rayleigh spectrum; black filled dots show the experimental data, and the red line shows the fit using an excitonic model [52]. Bottom: REPs of RBLMs, both in phase (blue open circles) and out of phase (black open diamonds), and corresponding fits using an excitonic model [52] (blue and black lines, respectively). The PLE spectrum fit gives the maximum of emission at excitation energies of $1.541 \mathrm{eV}\left(S_{22}^{i}\right.$ and $\left.M_{11+}^{o}\right)$ and $1.42 \mathrm{eV}\left(M_{11-}^{o}\right)$. The fits of the Rayleigh spectrum (REPs) give the energies of the transitions at $1.443(1.434)$ and $1.552(1.545) \mathrm{eV}$, respectively.

\section{DISCUSSION}

We first discuss the shifts of the transition energies compared to that of SWNTs. As summarized in Table I, the $S_{11}^{i}$ measured here are redshifted compared to the $S_{11}$ of the corresponding SWNTs [40] by an amplitude varying from -20 to $-100 \mathrm{meV}$. Such shifts were already observed for higher-order transitions $[6,7,35]$ by experiments performed on individual index-identified DWNTs. These shifts were mainly attributed to the electronic coupling between the incommensurate inner and outer tubes $[7,8]$ and to the effect of the interlayer dielectric screening of Coulomb interactions on the optical transitions in DWNTs [7,22]. The magnitudes of the energy shifts have been shown to depend sensitively on the specific optical transition and on the inner- and outer-tube species [7], which explains the tube-to-tube variations reported here. Furthermore, the changes in the exciton binding energy could have an additional impact on the lowest transition energies [22], but they still need to be evaluated.

Direct comparisons of the extracted PLE spectra, Rayleigh spectra, and RBLM REPs for the $(10,6) @(14,13)$ and $(14,1) @(15,12)$ DWNTs are displayed in Figs. 3(b) and 4(c), respectively. Small redshifts ( -5 to $-20 \mathrm{meV}$ ) are observed in the PLE spectra compared to RBLM REPs and Rayleigh spectra. Such shifts have already been observed in SWNTs and were attributed to the energy difference between the localized exciton dominating the PLE spectrum and the free exciton giving rise to the REP [44].

For all four investigated DWNTs, the ISCT PL signal is low, as highlighted, for example, by the comparison with the $2 G+2 D$ combination mode intensity in Fig. 2(c). Our results

TABLE I. Comparison of first excitonic transition energies of the measured DWNTs $\left(S_{11}^{i}\right)$ and of corresponding SWNTs $\left(S_{11}\right.$, from [40]).

\begin{tabular}{lcccc}
\hline \hline & \multicolumn{4}{c}{ DWNT } \\
\cline { 2 - 5 } & $(8,4) @(18,2)$ & $(7,6) @(16,6)$ & $(10,6) @(14,13)$ & $(14,1) @(15,12)$ \\
\hline$S_{11}^{i}(\mathrm{eV})$ & 1.128 & 1.058 & 0.828 & 0.792 \\
$S_{11}(\mathrm{eV})$ & 1.150 & 1.130 & 0.926 & 0.869 \\
Difference $(\mathrm{meV})$ & -22 & -72 & -98 & -78 \\
\hline \hline
\end{tabular}


suggest that the strong PL signal measured in different investigations performed on ensembles of DWNTs may clearly originate from SWNTs present in the samples [14,15,17-19,45].

In the perspective of possible optoelectronics developments, the PL QY, which is defined as the ratio of the number of photons emitted to the number of photons absorbed, appears to be a key parameter. In order to estimate the quantum yield of the studied DWNTs, we first performed a calibration of our experimental setup to determine the number of photons corresponding to one count detected. To do so, we first placed a silicon wafer under the microscope, focused on it with a laser with a wavelength of $950 \mathrm{~nm}$, and measured the reflected power at the exit of the microscope using a power meter. We then inserted into the laser path at the entrance of the microscope a series of calibrated neutral density filters in order to attenuate the laser sufficiently enough to record its signal with the InGaAs detector without saturating it. We thus determined, that $320 \pm 30$ photons exiting the microscope are required to accumulate 1 count on the detector. This number compares reasonably well with a simple estimate based on the manufacturers' specifications of the different elements of the setup, which gives 200 photons per count. By taking into account the transmissions of different elements of the microscope and the difference of the reflection efficiency of the spectrometer grating, we end up with 520 and 880 photons/count at $1.3 \mathrm{eV}(1100 \mathrm{~nm})$ and $0.8 \mathrm{eV}$ $(1550 \mathrm{~nm})$, respectively. The detector quantum efficiency can be considered constant in this range. Taking the solid angle of the collection of the microscope objective (numerical aperture of 0.95) into account as well, we can extract from the recorded PL spectra the number of emitted photons by summing the signal on all detector pixels.

In order to estimate the number of absorbed photons, (i) the absolute absorption cross section is taken as $0.6 \mathrm{~nm}^{2} / \mathrm{nm}$ per excited transition as measured on SWNTs [46] and on DWNTs [47], (ii) the laser spot size is measured by Raman mapping across a nanotube, and (iii) the impinging laser power is measured under the microscope objective. This procedure gives values of the QY of $5 \times 10^{-6}$ for the $(10,6) @(14,13)$ DWNT excited at $S_{22}^{i}$ and $3 \times 10^{-6}\left(2 \times 10^{-6}\right)$ for the $(14,1) @(15,12)$ DWNT excited at $S_{22}^{i}+M_{11+}^{o}\left(M_{11-}^{o}\right)$. Additionally, for the $(8,4) @(18,2)$ DWNT, we measured directly its extinction at $2.21 \mathrm{eV}(561 \mathrm{~nm})$, which is $4.9( \pm 0.5) \times 10^{-4}$ (Fig. 5). The extinction and the measurement of the impinging laser power give direct access to the number of photons absorbed by the DWNT during the measurement of its PL spectrum. Thus, we can estimate, with a direct measurement of its optical absorption, the QY of the $(8,4) @(18,2)$ DWNT excited at $2.21 \mathrm{eV}$ as $3.3( \pm 0.9) \times 10^{-6}$.

The value of the QY of the ISCT PL is thus found for the three investigated DWNTs, independent of the outer-tube SC or $\mathrm{M}$ nature, to be of the order of $10^{-6}$, i.e., four orders of magnitude lower than in SWNTs [48], in agreement with the conclusion of Ref. [20]. This weak QY can be understood in terms of (i) ultrafast exciton transfer from the inner to outer tube as suggested by femtosecond time-resolved luminescence measurements [21] and time-domain ab initio simulations [25] and (ii) charge transfer from outer to inner tubes [26,27]. Regarding the second effect, the observation of a rather strong electronic Raman scattering signal and broad component in

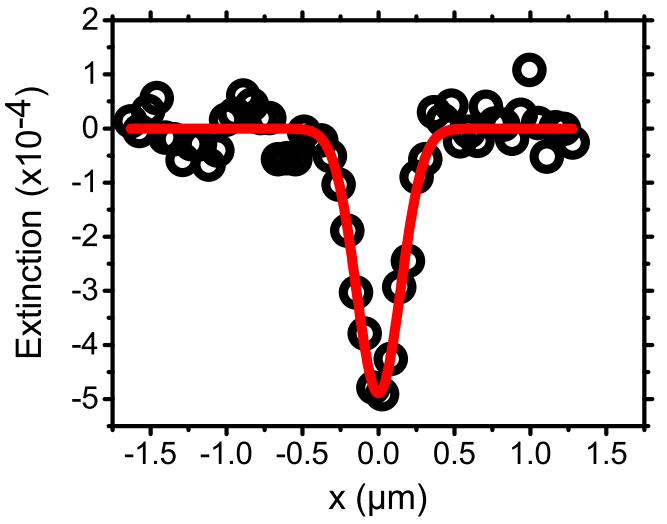

FIG. 5. Extinction, i.e., the relative change in the laser transmission signal $\Delta T / T_{0}$, measured by placing a photodiode underneath the sample and using it to record the transmitted laser power as a function of the $561+\mathrm{nm}$ laser position while scanning it across the $(8,4) @(18,2)$ DWNT (located at $x=0)$ : data are shown by black open circles, and the corresponding fit with a Gaussian function is shown by the red line.

the $G$ band in the $(14,1) @(15,12)$ DWNT [36] indicates that the doping level of this tube is, at most, of the order of 0.001 charge/atom [49,50]. Even if we cannot evaluate more precisely this doping, a recent study on DWNTs filled by linear carbon chains [27] has shown that balancing the intrinsic charge transfer between the outer tube and ISCT leads to a maximum increase of one order of magnitude of the PL signal. We thus infer that the main source of quenching would be the decay of the exciton through the lower-energy states of the outer tube compared to $S_{11}^{i}$ [25]. As discussed in Ref. [25], the detailed dynamics of exciton transfer in DWNTs is a complex and intricate process. Indeed, Postupna et al. [25] have shown that the exciton relaxation pathways are different for different DWNTs and that their dynamics can depend significantly on the initial system configuration. Furthermore, their work revealed that the detailed mechanism appears to go beyond the level alignment picture and involve geometry fluctuations and optically dark states. Nevertheless, simulated intertube transfer [25] and time-resolved luminescence experiments [21] agree for a transfer time of the order of $100 \mathrm{fs}$, which is in agreement with the low QY reported here. Regarding the observed $(14,1)$ ISCT PL upon excitation of the $(15,12)$ metallic outer tube, this transfer time has to be compared with the dynamics of photocarriers in metallic nanotubes. Hertel et al. [51] have shown that, for an excitation in the near infrared, the carriers in metallic tubes relax to the Fermi energy by electron-electron scattering within $\approx 50 \mathrm{fs}$. The two phenomena (intertube exciton transfer and photocarrier relaxation in metallic tubes) then appear to occur on very close time scales, which can explain our observation. Another possibility would be a significant mixing of energy levels with some delocalization between the tubes that could occur due to atomic motions at room temperature, as suggested by Postupna et al. [25]. We believe, however, that the first explanation is the most reasonable one given the magnitude of the effect and the fact that the Raman signatures remain unaffected. 


\section{CONCLUSION}

In conclusion, our results derived from combined TEM, Rayleigh spectroscopy, Raman spectroscopy, and PL experiments performed on individual freestanding DWNTs give unambiguous evidence of ISCT PL, which is independent of the SC or M nature of the outer tubes. Our results definitively close the debate about the existence of ISCT luminescence in DWNTs. The PL QY of ISCT in DWNTs is found to be low. It was estimated for three DWNTs as a few $10^{-6}$, i.e., four orders of magnitude lower than in freestanding SWNTs. This result is mainly attributed to ultrafast exciton transfer from the inner to outer tube. The investigation of outer-tube PL upon ISCT excitation could be a way to fully address this point. Furthermore, we have shown that it was possible to measure the PL signal of the $(14,1)$ ISCT through the excitation of the $M_{11-}^{o}$ optical transition of the $(15,12)$ outer tube. Moreover, the ISCT PL QY is found in this case to be comparable to the one estimated upon excitation of the ISCT $S_{22}^{i}$. More generally, this opens the question of the possibility of an exciton transfer occurring from the outer tube to the ISCT, i.e., in the opposite way to that usually observed.

\section{ACKNOWLEDGMENTS}

The authors thank J.-R. Huntzinger for helpful discussions. D.I.L. and S.B.R. gratefully acknowledge financial support from the Russian Science Foundation (Grant No. 15-12-10004). R.P., J.-L.S., A.-A.Z., and M.P. acknowledge financial support from the ANR GAMBIT project, Grant No. ANR-13-BS10-0014 of the French Agence Nationale de la Recherche, and the International Project of Scientific Cooperation with Vietnam (CNRS PICS 6457). V.C.N. acknowledges financial support from the Vietnamese National Foundation for Science and Technology Development (Grant No.103.99-2016.19). R.A. gratefully acknowledges the project "Construyendo Europa desde Aragon" 2014-2020 (Grant No. E/26) and the support from the Spanish Ministry of Economy and Competitiveness (MINECO) through Project Grant No. MAT2016-79776-P (AEI/FEDER, UE). The research leading to these results has received funding from the EU H2020 programs ETN Grant Agreement No. 642742. The TEM studies were conducted at the Laboratorio de Microscopías Avanzadas, Instituto de Nanociencia de Aragón, Universidad de Zaragoza, Spain. The government of Aragon and the European Social Fund are gratefully acknowledged.
[1] R. Pfeiffer, T. Pichler, Y. A. Kim, and H. Kuzmany, Top. Appl. Phys. 111, 495 (2008).

[2] R. Pfeiffer, F. Simon, H. Kuzmany, and V. N. Popov, Phys. Rev. B 72, 161404 (2005).

[3] D. Christofilos, J. Arvanitidis, G. A. Kourouklis, S. Ves, T. Takenobu, Y. Iwasa, and H. Kataura, Phys. Rev. B 76, 113402 (2007).

[4] K. Liu, X. Hong, M. Wu, F. Xiao, W. Wang, X. Bai, J. W. Ager, S. Aloni, A. Zettl, E. Wang, and F. Wang, Nat. Commun. 4, 1375 (2013).

[5] T. C. Hirschmann, P. T. Araujo, H. Muramatsu, J. F. RodriguezNieva, M. Seifert, K. Nielsch, Y. A. Kim, and M. S. Dresselhaus, ACS Nano 8, 1330 (2014).

[6] D. I. Levshov, T. Michel, R. Arenal, H. N. Tran, T. X. Than, M. Paillet, Y. I. Yuzyuk, and J.-L. Sauvajol, J. Phys. Chem. C 119, 23196 (2015)

[7] K. Liu, C. Jin, X. Hong, J. Kim, A. Zettl, E. Wang, and F. Wang, Nat. Phys. 10, 737 (2014).

[8] M. Koshino, P. Moon, and Y.-W. Son, Phys. Rev. B 91, 035405 (2015).

[9] A. Ghedjatti, Y. Magnin, F. Fossard, G. Wang, H. Amara, E. Flahaut, J.-S. Lauret, and A. Loiseau, ACS Nano 11, 4840 (2017).

[10] P. Avouris, M. Freitag, and V. Perebeinos, Nat. Photonics 2, 341 (2008).

[11] A. A. Green and M. C. Hersam, Nat. Nanotechnol. 4, 64 (2009).

[12] T. Sugai, H. Yoshida, T. Shimada, T. Okazaki, H. Shinohara, and S. Bandow, Nano Lett. 3, 769 (2003)

[13] A. H. Brozena, J. Moskowitz, B. Shao, S. Deng, H. Liao, K. J. Gaskell, and Y. Wang, J. Am. Chem. Soc. 132, 3932 (2010).

[14] T. Hertel, A. Hagen, V. Talalaev, K. Arnold, F. Hennrich, M. Kappes, S. Rosenthal, J. McBride, H. Ulbricht, and E. Flahaut, Nano Lett. 5, 511 (2005).
[15] N. Kishi, S. Kikuchi, P. Ramesh, T. Sugai, Y. Watanabe, and H. Shinohara, J. Phys. Chem. B 110, 24816 (2006).

[16] K. Iakoubovskii, N. Minami, S. Kazaoui, T. Ueno, Y. Miyata, K. Yanagi, H. Kataura, S. Ohshima, and T. Saito, J. Phys. Chem. B 110, 17420 (2006).

[17] H. Muramatsu, T. Hayashi, Y. A. Kim, D. Shimamoto, M. Endo, V. Meunier, B. G. Sumpter, M. Terrones, and M. S. Dresselhaus, Small 5, 2678 (2009).

[18] J. H. Kim, M. Kataoka, D. Shimamoto, H. Muramatsu, Y. C. Jung, T. Hayashi, Y. A. Kim, M. Endo, J. S. Park, R. Saito, M. Terrones, and M. S. Dresselhaus, ACS Nano 4, 1060 (2010).

[19] S. Yang, A. N. Parks, S. A. Saba, P. L. Ferguson, and J. Liu, Nano Lett. 11, 4405 (2011).

[20] D. A. Tsyboulski, Y. Hou, N. Fakhri, S. Ghosh, R. Zhang, S. M. Bachilo, M. Pasquali, L. Chen, J. Liu, and R. B. Weisman, Nano Lett. 9, 3282 (2009).

[21] T. Koyama, Y. Asada, N. Hikosaka, Y. Miyata, H. Shinohara, and A. Nakamura, ACS Nano 5, 5881 (2011).

[22] Y. Tomio, H. Suzuura, and T. Ando, Phys. Rev. B 85, 085411 (2012).

[23] T. Okazaki, S. Bandow, G. Tamura, Y. Fujita, K. Iakoubovskii, S. Kazaoui, N. Minami, T. Saito, K. Suenaga, and S. Iijima, Phys. Rev. B 74, 153404 (2006).

[24] C. Shen, A. H. Brozena, and Y. Wang, Nanoscale 3, 503 (2011)

[25] O. Postupna, R. Long, and O. V. Prezhdo, J. Phys. Chem. C 119, 12088 (2015)

[26] V. Zolyomi, J. Koltai, A. Rusznyak, J. Kurti, A. Gali, F. Simon, H. Kuzmany, A. Szabados, and P. R. Surjan, Phys. Rev. B 77, 245403 (2008).

[27] P. Rohringer, L. Shi, P. Ayala, and T. Pichler, Adv. Funct. Mater. 26, 4874 (2016).

[28] A. A. Green and M. C. Hersam, ACS Nano 5, 1459 (2011). 
[29] P. Rohringer, L. Shi, X. Liu, K. Yanagi, and T. Pichler, Carbon 74, 282 (2014).

[30] J. C. Meyer, M. Paillet, T. Michel, A. Moréac, A. Neumann, G. S. Duesberg, S. Roth, and J.-L. Sauvajol, Phys. Rev. Lett. 95, 217401 (2005).

[31] M. Paillet, T. Michel, J. C. Meyer, V. N. Popov, L. Henrard, S. Roth, and J.-L. Sauvajol, Phys. Rev. Lett. 96, 257401 (2006).

[32] M. Y. Sfeir, T. Beetz, F. Wang, L. Huang, X. M. H. Huang, M. Huang, J. Hone, S. P. O’Brien, J. A. Misewich, T. F. Heinz, L. Wu, Y. Zhu, and L. E. Brus, Science 312, 554 (2006).

[33] K. Liu, J. Deslippe, F. Xiao, R. B. Capaz, X. Hong, S. Aloni, A. Zettl, W. Wang, X. Bai, S. G. Louie, E. Wang, and F. Wang, Nat. Nanotechnol. 7, 325 (2012).

[34] D. Levshov, T. X. Than, R. Arenal, V. N. Popov, R. Parret, M. Paillet, V. Jourdain, A. A. Zahab, T. Michel, Y. I. Yuzyuk, and J.-L. L. Sauvajol, Nano Lett. 11, 4800 (2011).

[35] S. Zhao, T. Kitagawa, Y. Miyauchi, K. Matsuda, H. Shinohara, and R. Kitaura, Nano Res. 7, 1548 (2014).

[36] See Supplemental Material at http://link.aps.org/supplemental/ 10.1103/PhysRevB.96.195410, which includes Refs. [53-55], for details of the sample fabrication method and experimental measurement procedures; a detailed characterization and indexation of all four individual DWNTs, based on ED, HRTEM, and Rayleigh and Raman spectroscopies; PL spectra of the $(7,6) @(16,6)$ DWNT measured at $693 \mathrm{~nm}(1.79 \mathrm{eV})$ and $700 \mathrm{~nm}$ $(1.77 \mathrm{eV})$ and plotted as a function of Raman shift (with the $2 G+2 D$ Raman peak indicated in the figure); PL spectra of $(14,1) @(15,12)$ DWNT; Raman spectra of individual SC@M $(14,1) @(15,12)$ DWNTs, measured at $776 \mathrm{~nm}(1.60 \mathrm{eV}), 850 \mathrm{~nm}$ $(1.46 \mathrm{eV})$, and $457 \mathrm{~nm}(2.71 \mathrm{eV})$, showing electronic Raman peaks; and a resonant Raman excitation map and resonant Raman excitation profiles of the $(14,1) @(15,12)$ DWNT in the $G$-mode range.

[37] K. Hirahara, M. Kociak, S. Bandow, T. Nakahira, K. Itoh, Y. Saito, and S. Iijima, Phys. Rev. B 73, 195420 (2006).

[38] D. I. Levshov, H. N. Tran, M. Paillet, R. Arenal, X. T. Than, A. A. Zahab, Y. I. Yuzyuk, J.-L. Sauvajol, and T. Michel, Carbon 114, 141 (2017).
[39] M. Paillet, F. Meunier, M. Verhaegen, S. Blais-Ouellette, and R. Martel, Rev. Sci. Instrum. 81, 053111 (2010).

[40] J. Lefebvre and P. Finnie, Phys. Rev. Lett. 98, 167406 (2007).

[41] V. N. Popov and L. Henrard, Phys. Rev. B 65, 235415 (2002).

[42] V. N. Popov, L. Henrard, and P. Lambin, Phys. Rev. B 72, 035436 (2005).

[43] M. Machon, S. Reich, H. Telg, J. Maultzsch, P. Ordejon, and C. Thomsen, Phys. Rev. B 71, 035416 (2005).

[44] M. Steiner, M. Freitag, J. C. Tsang, V. Perebeinos, A. A. Bol, A. V. Failla, and P. Avouris, Appl. Phys. A 96, 271 (2009).

[45] K. Iakoubovskii, N. Minami, T. Ueno, S. Kazaoui, and H. Kataura, J. Phys. Chem. C 112, 11194 (2008).

[46] J.-C. Blancon, M. Paillet, H. N. Tran, X. T. Than, S. A. Guebrou, A. Ayari, A. San Miguel, N.-M. Phan, A.-A. Zahab, J.-L. Sauvajol, N. Del Fatti, and F. Vallée, Nat. Commun. 4, 2542 (2013).

[47] J.-C. Blancon, Ph.D. thesis, University Claude Bernard Lyon I, 2013.

[48] J. Lefebvre, D. G. Austing, J. Bond, and P. Finnie, Nano Lett. 6, 1603 (2006).

[49] H. Farhat, S. Berciaud, M. Kalbac, R. Saito, T. F. Heinz, M. S. Dresselhaus, and J. Kong, Phys. Rev. Lett. 107, 157401 (2011).

[50] H. Farhat, H. Son, Ge. G. Samsonidze, S. Reich, M. S. Dresselhaus, and J. Kong, Phys. Rev. Lett. 99, 145506 (2007).

[51] T. Hertel, R. Fasel, and G. Moos, Appl. Phys. A 75, 449 (2002).

[52] H. N. Tran, J.-C. Blancon, J.-R. Huntzinger, R. Arenal, V. N. Popov, A. A. Zahab, A. Ayari, A. San-Miguel, F. Vallée, N. Del Fatti, J.-L. Sauvajol, and M. Paillet, Phys. Rev. B 94, 075430 (2016).

[53] S. Berciaud, V. V. Deshpande, R. Caldwell, Y. Miyauchi, C. Voisin, P. Kim, J. Hone, and T. F. Heinz, Phys. Status Solidi 249, 2436 (2012).

[54] F. Wang, W. Liu, Y. Wu, M. Y. Sfeir, L. Huang, J. Hone, S. O'Brien, L. E. Brus, T. F. Heinz, and Y. R. Shen, Phys. Rev. Lett. 98, 047402 (2007).

[55] H. N. Tran, J.-C. Blancon, R. Arenal, R. Parret, A. A. Zahab, A. Ayari, F. Vallée, N. Del Fatti, J.-L. Sauvajol, and M. Paillet, Phys. Rev. B 95, 205411 (2017). 